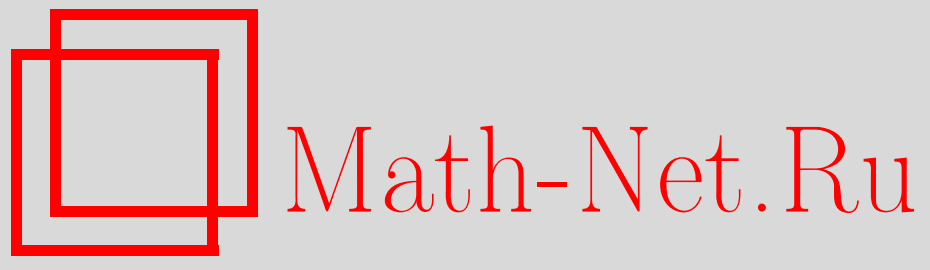

P. Carr, M. Schröder, Bessel processes, the integral of geometric Brownian motion, and Asian options, Теория вероятн. и ее примен., 2003, том 48, выпуск $3,503-533$

DOI: https://doi.org/10.4213/tvp268

Использование Общероссийского математического портала MathNet.Ru подразумевает, что вы прочитали и согласны с пользовательским соглашением

http://www . mathnet.ru/rus/agreement

Параметры загрузки:

IP : 52.23 .180 .231

26 апреля 2023 г., 09:08:43

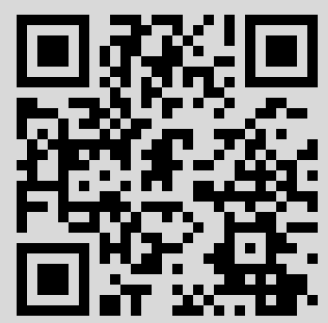




\section{BESSEL PROCESSES, THE INTEGRAL OF GEOMETRIC BROWNIAN MOTION, AND ASIAN OPTIONS}

Статья возникла под влиянием вопросов о средних значениях случайных процессов, возникающих в финансовой математике в связи с отысканием цены так называемых Азиатских опционов.

Начиная с работы [29], эти вопросы об экспоненциальных функционалах от броуновского движения изучались в терминах процессов Бесселя с помощью теории Хартмана-Ватсона [27], и результаты о преобразованиях Лапласа, полученные в [12], явились заметным продвижением в оценивании Азиатских опционов. $\mathrm{K}$ сожалению, возник ряд затруднений с ключевыми результатами последней статьи, которые и обсуждаются в данной статье. Одно из этих затруднений носит принципиальный характер и проистекает из самого подхода Хартмана-Ватсона: этот подход, как правило, применим без модификаций, только если он не сталкивается с процессами Бесселя с отрицательными индексами. В настоящей статье мы развиваем три подхода к преодолению этих трудностей, в частности, по-новому комбинируя стохастические методы и комплексный анализ, и обсуждаем их следствия для нахождения цены Азиатских опционов.

Ключевые слова и фразы: опционы Азиатского типа, интеграл от геометрического броуновского движения, процессы Бесселя, преобразование Лапласа, методы комплексного анализа в стохастике.

1. Introduction. This paper addresses questions about exponential functionals of Brownian motion and the integral of geometric Brownian motion in particular. These questions reduce to the study of the quadratic variation processes $A^{(\nu)}$ of geometric Brownian motion which for any real drift $\nu$ are explicitly given by the integrals over time

$$
A_{t}^{(\nu)}=\int_{0}^{t} e^{2\left(\nu w+B_{w}\right)} d w, \quad t \in[0, \infty),
$$

with $B$ a standard Brownian motion. These processes have both a surprisingly rich theory and manifold applications ranging from the physics of

* Analytic Research at Bloomberg (ARB), 499 Park Avenue, New York, NY 10022 and Courant Institute, NYU, 251 Mercer Street, New York NY 10012, USA; e-mail: pcarr@nyc.rr.com

** Keplerstraße 30, D-69469 Weinheim, Germany; e-mail: schroeder@math.unimannheim.de 
random media to mathematical finance and insurance. In fact, the insurance motivated study of certain perpetuities in [8] seems to have initiated this line of research. Here, the above integrals over the whole time axis are considered and shown to be distributed as the reciprocals of certain gamma variables. Drawing on his probabilistic interpretation of the Hartman-Watson identities in [27], Yor was able to extend this work and to determine the law of the processes $A^{(\nu)}$ in [29]. This approach, using the Laplace transform and based on Bessel processes, has been found by Yor to open many surprising vistas on the processes $A^{(\nu)}$ and their applications, see in particular [32]. The interest in these processes $A^{(\nu)}$ is partially due to their importance in mathematical finance, in particular for understanding the so-called Asian options.

Asian options are widely used financial derivatives. As discussed in Part I of the paper, these options provide in general nonlinear payoffs on the arithmetic average of the price of an underlying asset. A common objective in their valuation is to derive an explicit expression for a certain functional of $A^{(\nu)}$. The pursuit of this objective has evolved over the last fifteen years as an interplay between theoretical and computational perspectives, see [20] or [9] for instance. Yor's work in [29] clarified the structure of the BlackScholes prices of Asian options by expressing them as certain triple integrals. In contrast to this result, however, it was the Laplace transform approach of [12], also based on Hartman-Watson theory, which had far reaching consequences for the way Asian option valuation is seen today, and on which we focus here from Part II onwards. This is essentially because a very explicit expression in terms of Kummer's confluent hypergeometric function resulted from this approach for what has been regarded as the Laplace transform of the value of such options. From a numerical point of view, this expression has proved to be amenable to computation by numerical inversion, see [11] as a recent example. And it seems fair to say that this has lead financial mathematics to a new interest in developing and applying these techniques.

Unfortunately, some difficulties with this Laplace transform approach to valuing Asian options have emerged. First, it turned out that the Laplace transforms computed in [12] are not those of the Asian option's value. This appeared to empty the relevance of this result for the finance application proper. Luckily, it turned out that there is a reduction of the original problem of valuing Asian options to the one considered in [12]. All of this is discussed in Part II of the paper.

A difficulty of a more serious and more principal nature, however, originates with the Hartman-Watson approach on which the Laplace transform computations of [12] are based. Its idea is to analyze $A^{(\nu)}$ using Bessel processes of indices $\nu$, and its applicability has limits if $\nu$ is negative because of the pathologies which Bessel processes of such negative indices develop. Together with background material about Bessel processes, we have thus given 
in Part III a new exposition of the analysis in [12], which explicitly takes care of the nonnegativity restriction on the index $\nu$. Hereby, we have been encouraged by the kind support of Yor, and we have tried to incorporate his kind tutorials and suggestions. In terms of financial mathematics, our extension to negative $\nu$ in fact extends the analysis of Asian options from the zero dividend situation originally considered in [12] to one of general real dividend yields, and thus to one with general real risk-neutral drifts. In this setting, the condition that $\nu$ is not negative transcribes into the postulate that risk-neutral drift is not less than half the squared volatility. Unfortunately, this lower bound on the drift restricts the financial applicability of the results. In fact, the greater is the volatility, the greater is the range of parameters in which the nonnegativity condition on $\nu$ is violated and in which the approach does not give the Laplace transforms. Unfortunately, it is precisely due to high volatility of the underlying asset that Asian options are used in the first place.

Thus, the third contribution of our paper is to remove these restrictions by showing the existence of the desired Laplace transforms. In fact, we develop three ways for removing these restrictions. More precisely, we develop three principal ways of coping with the difficulties caused by Bessel processes of negative indices in the Hartman-Watson approach. This can be seen as the main mathematical contribution of the paper. The approaches of Parts IV and VI are based on an analysis of Bessel processes in the spirit of Yor. Our first approach extends the [12] analysis to the missing cases using deeper properties of Bessel processes of negative indices. Our second approach gives a new uniform proof using zero index Bessel processes only. Our third approach of Part V extends that of [2] and merges stochastic with complex analytic techniques. This appears to be a rather novel and promising line of attack as we are able to largely dispense with Bessel processes and focus instead on Brownian motion.

Apart from all this, our extension of the Laplace transform approach of [12] has made possible advances in valuing Asian options, some of which are sketched in Part VII. Hence, it may be fair to say that the Laplace transform approach of [12] has proved to be a rich source for new results and insights in both finance and mathematics.

\section{Part I. Prologue}

2. Black-Scholes modelling. The results to be discussed originate from the so-called risk-neutral approach to the valuation of contingent claims. General equilibrium treatments for this and other notions developed for the analysis of financial markets and instruments are in [6], [7], [13, Chapters 1-4], for instance. This analysis is based on models of security markets, and this section aims to recall the most fundamental of these, the Black-Scholes model of security markets. 
In fact, we need only that particular case of the Black-Scholes model, where there are only two securities, and the understanding is that these are traded on markets, where their prices are determined by equating demand and supply. First, there is a riskless security, a bond, whose price $\beta$ grows at the continuously compounding positive interest rate $r$, i.e., for which we have $\beta_{t}=\exp (r t)$ at any time $t$ in $[0, \infty)$. Then, there is a risky security. The fundamental idea is that all uncertainties affecting its price $S$ yield a certain probability space. In fact, consider for this a complete probability space equipped with the standard filtration of a standard Brownian motion on the time set $[0, \infty)$. Giving expression to the fact that $S$ comes as an equilibrium price, we have the risk-neutral measure $\mathbf{Q}$ on this filtered space, a probability measure equivalent to the given one. And with $B$ any standard Q-Brownian motion, the exact modelling then is that $S$ is the strong solution of the following stochastic differential equation:

$$
d S_{t}=\varpi S_{t} d t+\sigma S_{t} d B_{t}, \quad t \in[0, \infty),
$$

or equivalently using Itô calculus,

$$
S_{t}=S_{0} e^{\left(\varpi-\sigma^{2} / 2\right) t+\sigma B_{t}} \quad t \in[0, \infty) .
$$

The positive constant $\sigma$ is the volatility of $S$. The specific form of the otherwise arbitrary constant $\varpi$ depends on the nature of the security modelled which could be a stock, a currency, a commodity etc. For example, if $S$ is the price of a stock paying a dividend continuously so as to have constant dividend yield $\delta$, then we have $\varpi=r-\delta$.

3. Asian options and their equilibrium pricing. In the BlackScholes framework of $\S 2$, fix any time $t_{0}$ and consider the process $J$ given for any time $t$ by

$$
J(t)=\int_{t_{0}}^{t} S_{u} d u
$$

The arithmetic-average Asian option written at time $t_{0}$ with maturity $T$ and strike price $K$ is then the stochastic process on the closed time interval from $t_{0}$ to $T$ paying

$$
\left(\frac{J(T)}{T-t_{0}}-K\right)^{+}=\max \left\{0, \frac{J(T)}{T-t_{0}}-K\right\}
$$

at time $T$ and paying nothing at all other times. As such it is a contingent claim on the time interval from $t_{0}$ to $T$ with payoff $\left(J(T) /\left(T-t_{0}\right)-K\right)^{+}$.

It is one of the fundamental insights that in the equilibrium framework of the Black-Scholes model, any such contingent claim on a risky security has an equilibrium price which is equal to the discounted expectation of its payoff with respect to the risk-neutral measure conditional on today's information, 
see $[6, \S 22 \mathrm{~K},(47)],[7, \S 8 \mathrm{~A}]$ or $[16$, Corollary 5.1.1], for instance. Applying this arbitrage pricing principle, the price $C_{t}$ of the Asian option at any time $t$ between $t_{0}$ and $T$ is given by the discounted $\mathbf{Q}$-expectation conditional on the information $\mathscr{F}_{t}$ available at time $t$ :

$$
C_{t}=e^{-r(T-t)} \mathbf{E}^{\mathrm{Q}}\left[\left(\frac{J(T)}{T-t_{0}}-K\right)^{+} \mid \mathscr{F}_{t}\right] .
$$

However, following $[12, \S 3.2]$, we do not focus on this price, but instead we normalize the valuation problem as follows. On factoring out the reciprocal of the length $T-t_{0}$ of the time period, we split the integral $J(T)$ into two integrals, one of which is deterministic by time $t$ and the other of which is random. We then couple the deterministic integral with the new strike. For the random integral, we restart the Brownian motion driving the underlying at time $t$, and then using the scaling property of Brownian motion, we change time so as to normalize its coefficient in the new time scale to two. The precise result is the factorization

$$
C_{t}=\frac{e^{-r(T-t)}}{T-t_{0}} \frac{4 S_{t}}{\sigma^{2}} C^{(\nu)}(h, q),
$$

which reduces the general valuation problem to computing $C^{(\nu)}(h, q)=$ $\mathbf{E}^{\mathbf{Q}}\left[\left(A_{h}^{(\nu)}-q\right)^{+}\right]$, the normalized time-t price of the Asian option. Herein, $A^{(\nu)}$ is Yor's process

$$
A_{h}^{(\nu)}=\int_{0}^{h} e^{2\left(B_{w}+\nu w\right)} d w
$$

and the normalized parameters are as follows: $\nu=2 \varpi / \sigma^{2}-1, h=\sigma^{2}(T-$ t) $/ 4, q=k h+q^{*}$, where $k=K / S_{t}, q^{*}=q^{*}(t)=\sigma^{2}\left(4 S_{t}\right)^{-1}\left(K\left(t-t_{0}\right)-\right.$ $\left.\int_{t_{0}}^{t} S_{u} d u\right)$.

To interpret these quantities, $\nu$ is the normalized adjusted interest rate, $h$ is the normalized time to maturity, which is nonnegative, and $q$ is the normalized strike price. On a conceptual level, notice that valuing any Asian option in this way is reduced to computing a single function $C^{(\nu)}$, and that a similar notion of normalized hedging of Asian options can be developed along these lines. On a structural level, moreover notice how $q$ becomes affine linear in the time variable $h$ with coefficients $k$ and $q^{*}$ depending only on quantities known at time-t.

4. A first reduction of the normalized valuation. There is now a dichotomy in computing the normalized time-t price

$$
C^{(\nu)}(h, q)=\mathbf{E}^{\mathbf{Q}}\left[\left(A_{h}^{(\nu)}-q\right)^{+}\right]
$$

of the Asian option according to the normalized strike price $q$ being positive or not. Indeed, if $q$ is nonpositive, Asian options lose their option feature. Computing the values $C^{(\nu)}(h, q)$ is straightforward: 
Lemma. If $q \leqslant 0$, we have $C^{(\nu)}(h, q)=\mathbf{E}^{\mathbf{Q}}\left[A_{h}^{(\nu)}\right]-q$ with $\mathbf{E}^{\mathbf{Q}}\left[A_{h}^{(\nu)}\right]=$ $e^{2 h(\nu+1)}-1 /(2(\nu+1))$, for any real $\nu$, and it is thus sufficient to compute $C^{(\nu)}(h, q)$ if $q>0$.

This can be proved on applying Fubini's theorem, and the last expectation is seen to be analytic in $\nu$ with its value at $\nu=-1$ equal to $h$. It should be mentioned that formulas for all moments of $A^{(\nu)}$ have been derived at various instances over the last fifty years, see for example $\left[32, \S 2.4 .1,\left(4 . d^{\prime}\right)\right.$, p. 33 and Postscript \#3b), p. 54].

5. Yor's integral representation for Asian option values. A closed form for the normalized time-t prices $C^{(\nu)}$ of Asian options can be obtained as a consequence of Yor's triple integral representation [29, (6.e), p. 528]. Recall the latter is based on Yor's characterization of the law of $A^{(\nu)}$ in $[29,(6 . c)$, p. 527$]$ and so is eventually based on the Hartman-Watson theory of [27]. Furnishing a measure for the difficulty of computing normalized prices, the precise form of Yor's closed form is as follows.

Theorem. For any reals $h, q>0$ and $\nu$, we have

$$
C^{(\nu)}(h, q)=c_{\nu, h} \int_{0}^{\infty} x^{\nu} \int_{0}^{\infty} e^{-\left(1+x^{2}\right) y / 2}\left(\frac{1}{y}-q\right)^{+} \psi_{x y}(h) d y d x
$$

Herein the function $\psi_{a}$, for any positive real number $a$, is given by the following integral:

$$
\psi_{a}(h)=\int_{0}^{\infty} e^{-w^{2} /(2 h)} e^{-a \operatorname{ch}(w)} \operatorname{sh}(w) \sin \left(\frac{\pi}{h} w\right) d w
$$

for any $h>0$, and we abbreviate

$$
c_{\nu, h}=\frac{1}{\pi \sqrt{2 \pi^{3} h}} e^{\pi^{2} /(2 h)-\nu^{2} h / 2} .
$$

While Yor's formula seems to require $\nu$ to be bigger than at least minus one, it is valid for all real $\nu$. This is proved in $[24, \S 6]$.

One purpose of closed form expressions is to provide means for actually computing option prices. While Yor's results above are the key to many insights into the mathematical structure of $A^{(\nu)}$, Yor's formula of the Theorem has a number of structural difficulties in this regard. First, it involves three integrations with seemingly no further structure or further possibilities for simplification. Thus, methods for explicitly computing it will necessarily be rather complex. However, we have noticed an apparently even bigger obstacle to computability. For instance taking $t_{0}=0$ and $\varpi=r$ equal to $5 \%$, we 
compute the factors $c_{\nu, h}$ as follows:

\begin{tabular}{|c|c|c|c|}
\hline$c_{\nu, h}$ & $\sigma=20 \%$ & $\sigma=30 \%$ & $\sigma=40 \%$ \\
\hline$T=1$ year & $2.627 \times 10^{213}$ & $2.265 \times 10^{94}$ & $4.816 \times 10^{52}$ \\
\hline$T=6$ months & $7.686 \times 10^{427}$ & $5.717 \times 10^{189}$ & $2.583 \times 10^{106}$ \\
\hline
\end{tabular}

Table 1. $c_{\nu, h}$ as function of $T$ and $\sigma$.

As the examples of $\S 8$ or $\S 24$ will show, normalized prices of Asian options are not too big. Yor's formula thus expresses them as the product of a big number times a triple integral. The latter so has to be very small and must be computed with very high accuracies to get reasonably accurate results. The Laplace transform approach developed in [12] explicitly for the purpose of valuing Asian options was seen to offer a way out of these difficulties.

\section{Part II. Laplace transform results}

6. The Laplace transform in option valuation. Working with continuous functions on the nonnegative real line of at most exponential growth, the Laplace transform $\mathscr{L}(f)$ of any such function $f$ is defined by

$$
\mathscr{L}(f)(z)=\int_{0}^{\infty} e^{-z x} f(x) d x,
$$

for any complex number $z$ in a half-plane contained sufficiently deep within the complex right half-plane.

The connection of this notion with option valuation in general and Asian option valuation in particular is as follows. Fix any option type, like $\S 3$ 's European style Asian call option on a certain stock with price $S$. It will depend on a number of parameters, like strike price and maturity date. At a fixed point in time $t$, consider the family which consists of all options on the market with all such parameters fixed except maturity dates $M$. In the Asian option example thus consider the Asian options of all maturities available at time $t$ which have the same strike price. In this way regard maturity date $M$ as a real variable ranging from $t$ to infinity. The value of the option thus becomes a function of $M$.

However, it is normalized prices $C^{(\nu)}$ we have to consider for the Asian option. The normalizations of $\S 3$ in fact turn $C^{(\nu)}$ into a function of, in particular, normalized time to maturity. As a function of maturity date $M$ normalized time to maturity is explicitly given by $h(M)=\left(\sigma^{2} / 4\right)(M-t)$. With $M$ from $t$ to infinity, $h(M)$ thus ranges from 0 to infinity. The normalized price of the Asian option so becomes a function on the nonnegative real line. Call it $f_{A O}$ for the sake of emphasis. Recalling from $\S 3$ how the 
normalized strike price $q$ depends in an affine linear way on normalized time to maturity, we then have more precisely

$$
f_{A O}(x)=\mathbf{E}^{\mathbf{Q}}\left[\left(A_{x}^{(\nu)}-\left(k x+q^{*}\right)\right)^{\dagger}\right]
$$

for any nonnegative real $x$.

At this point we should signal a difficulty with [12] which we have noticed in Fall 1999. It is not the functions $f_{A O}$ this last paper is working with, but the functions $f_{G Y, a}$ given for any positive real $a$ by $f_{G Y, a}(x)=$ $\mathbf{E}^{\mathbf{Q}}\left[\left(A_{x}^{(\nu)}-a\right)^{+}\right]$for any nonnegative real $x$. Notice that the function $f_{A O}$ has a nonconstant strike price $x \mapsto k x+q^{*}$ whereas any function $f_{G Y, a}$ has the constant strike price $a$. So the value function of the Asian option $f_{A O}$ is different from any function $f_{G Y, a}$ which thus is the value function of a non-Asian option. Because of the injectivity of the Laplace transform on continuous functions, the Laplace transform $\mathscr{L}\left(f_{A O}\right)$ is different from any Laplace transform $\mathscr{L}\left(f_{G Y, a}\right)$ too. The problem at this point so is one of relevance of the mathematics for the finance application proper: is there a way of relating the valuation of Asian options to the valuation of the non-Asian options?

\section{Valuing Asian options using families of non-Asian options.} The basic idea is as follows. Try to reconstruct the normalized time- $t$ price

$$
C^{(\nu)}(h, q)=\left.\mathbf{E}^{\mathbf{Q}}\left[\left(A_{x}^{(\nu)}-\left(k x+q^{*}\right)\right)^{+}\right]\right|_{x=h}
$$

of the Asian option from a family of auxiliary functions whose single members are unrelated to the problem of valuing the Asian option but amenable to the [12] analysis. Given that the time dependency of the normalized strike price poses the problems, simply force this strike price to be constant. Thus arrive, for any positive real $a$, at $\S 6$ 's functions $f_{G Y, a}$ on the positive real line recalled to be given by $f_{G Y, a}(x)=\mathbf{E}^{\mathbf{Q}}\left[\left(A_{x}^{(\nu)}-a\right)^{+}\right]$for any positive real $x$. These are the functions considered in [12]. As we remarked in $\S 6$ they are the values of certain non-Asian options, and taken individually, they cannot be used to value the original Asian option. However, our finding is that as a whole they allow one to recover the normalized time- $t$ price. With the concepts of $\S 3$ this key reduction is made precise in our following lemma.

Lemma. If $q=k h+q^{*}$ is positive, computing the normalized time-t price $C^{(\nu)}(h, q)$ of the Asian option reduces to computing all $f_{G Y, a}$ with $a>0$. More precisely, $C^{(\nu)}(h, q)$ is obtained by choosing the function $f_{G Y, k h+q^{*}}$ and evaluating it at $h$.

A moment's reflection will convince the reader that this is true by construction, and meanwhile, the particular case of the Lemma we explained to Yor in May 2000 can be found in [32, p. 95-96]. Notice that our construction works in the more general situation, where functions of the form 
$h(y)=f(y, \varphi(y))$ with a known map $\varphi$ have to be computed: compute the functions $f$ and then intersect with the graph of $\varphi$ to get $h$. And to stress the main result again, as one consequence of this technique, we have at this point reduced valuing Asian options to valuing the family of all non-Asian options.

8. Laplace transforms of the non-Asian option values $f_{G Y, a}$. The key reduction of the preceding $\S 7$ shows the way to apply the [12] Laplace transform to value Asian options. Moreover, adopting the notation of $\S 3$, consider the family of all functions $f_{G Y, a}$ with $a>0$ that send any $x>0$ to

$$
f_{G Y, a}(x)=\mathbf{E}^{\mathbf{Q}}\left[\left(A_{x}^{(\nu)}-a\right)^{+}\right] .
$$

Recall from $\S 7$ that its single members are unrelated to valuing the Asian option, but that as a whole, the family allows reconstruction of the normalized time-t price $C^{(\nu)}(h, q)$ of the Asian option. As a first step in actually computing the $f_{G Y, a}$, try to compute their Laplace transforms $F_{G Y, a}$ given by

$$
F_{G Y, a}(z)=\int_{0}^{\infty} e^{-z x} f_{G Y, a}(x) d x=\mathscr{L}\left(f_{G Y, a}\right)(z) .
$$

Here the complex number $z$ is to be taken in a half-plane sufficiently deep within the right complex half-plane such that the integrals are finite. The function so obtained is analytic. The precise conditions under which these integrals are finite is part of our description of these generalized Geman-Yor Laplace transforms in the following theorem.

Theorem. If the normalized strike price $q$ is positive, the integrals $F_{G Y, a}$ are finite for any complex number $z$ with $\operatorname{Re}(z)>\max \{0,2(\nu+$ 1)\}, and we have

$$
F_{G Y, a}(z)=\frac{D_{\nu}(a, z)}{z(z-2(\nu+1))},
$$

where on choosing the principal branch of the logarithm

$$
D_{\nu}(a, z)=\frac{e^{-1 /(2 a)}}{a} \int_{0}^{\infty} e^{-x^{2} /(2 a)} x^{\nu+3} I_{\sqrt{2 z+\nu^{2}}}\left(\frac{x}{a}\right) d x .
$$

Herein $I_{\mu}$ is the modified Bessel function with complex order $\mu$, as discussed in [15, Chapter 5]. Generalizing [12, (3.9), p. 363], the integral $D_{\nu}(a, z)$ can be expressed using the confluent hypergeometric function $\Phi$ discussed in [15, Chapter 9] as follows.

Corollary. For any complex $z$ with $\operatorname{Re}(z)>\max \{0,2(\nu+1)\}$, we have

$$
\begin{aligned}
D_{\nu}(a, z)= & \Gamma\left(\frac{\nu+4+\mu(z)}{2}\right) \frac{l}{\Gamma(\mu(z)+1)} \\
& \times \Phi\left(\frac{\nu+4+\mu(z)}{2}, \mu(z)+1 ; \frac{1}{2 a}\right) \frac{(2 a)^{(\nu+2-\mu(z)) / 2}}{e^{1 /(2 a)}}
\end{aligned}
$$

on setting $\mu(z)=\sqrt{2 z+\nu^{2}}$. 
We again stress that these Laplace transforms are not those of the Asian option price, but rather the Laplace transforms of the prices of auxiliary options. To obtain Asian option prices, we have to invert these Laplace transforms and then proceed using $\S 7$ Lemma; formally speaking: $C^{(\nu)}(h, q)=\mathscr{L}^{-1}\left(F_{G Y, q}\right)(h)$. In full mathematical generality, analytic inversion has been achieved in [21], and we come back to this in $\S 23$. Numerical inversions have also been accomplished. For example, Fu, Madan and Wang [11] computed the following seven cases as reproduced in [9, Table 7.1]:

\begin{tabular}{|c|c|c|c|c|c|c|}
\hline Case & $r$ & $\sigma$ & $T$ & $S_{0}$ & $\nu$ & $2 C^{(\nu)}$ \\
\hline 1 & $2 \%$ & $10 \%$ & 1 & 2.0 & 3 & 0.056 \\
\hline 2 & $18 \%$ & $30 \%$ & 1 & 2.0 & 3 & 0.219 \\
\hline 3 & $1.25 \%$ & $25 \%$ & 2 & 2.0 & -0.6 & 0.172 \\
\hline 4 & $5 \%$ & $50 \%$ & 1 & 1.9 & -0.6 & 0.194 \\
\hline 5 & $5 \%$ & $50 \%$ & 1 & 2.0 & -0.6 & 0.247 \\
\hline 6 & $5 \%$ & $50 \%$ & 1 & 2.1 & -0.6 & 0.307 \\
\hline 7 & $5 \%$ & $50 \%$ & 2 & 2.0 & -0.6 & 0.352 \\
\hline
\end{tabular}

Table 2. Prices $2 C^{(\nu)}$ for $K=2.0$ and $t_{0}=t=0$ using numerical Laplace inversion.

Obtaining our two results proceeds in two steps. In a first probabilistic step, the arguments of [12] are adapted to compute the modified GemanYor transforms $F_{G Y, a}$. We give in Part III a new exposition of the argument incorporating the tutorials and kind suggestions of Yor. The key idea is to factorize the geometric Brownian motion of the underlying over a Bessel process of index $\nu$. Pertinent notions are discussed in $\S 9$. This makes time stochastic in such a way that Yor's process $A^{(\nu)}$ now takes the double role of both a stochastic clock and a control variable for the Asian option. At first sight, this appears to complicate the original valuation problem. However, this double role of $A^{(\nu)}$ is especially suited to the Laplace transform. Indeed, in contrast to the situation for the Asian option, the strike price $a$ of the nonAsian option with value function $f_{G Y, a}$ is independent of time. This makes it possible to reduce the computation of the Laplace transform $F_{G Y, a}$ to the following problem: obtain explicit expressions for the Bessel semigroup of index $\nu$ and for a certain conditional expectation involving first passage times of Yor's process $A^{(\nu)}$.

However, such results are available for both concepts only if the index $\nu$ is nonnegative, an assumption which is explicit in $[12, \S 2]$. This nonnegativity condition, however, translates into the condition that the risk-neutral drift is not less than half the squared volatility. Unfortunately, this places restrictions on the financial applicability of the result. For example, if volatil- 
ity is $30 \%$, the arguments of $[12, \S 3]$ are not valid if the difference between the risk-free rate and the dividend yield is less than $4.5 \%$, and then we do not have the Laplace transforms of the non-Asian options either. Worse yet, the greater is the volatility, the greater is this range of parameters in which we do not have the Laplace transforms. Unfortunately, it is precisely due to high volatility that Asian options are used in the first place.

We have been a bit disconcerted by these findings. Luckily, however, we found that in particular those results of Table 2, where $\nu$ is negative were reproduced in [9] using an alternative approach. And corroborating André Weil's [26, p. 457] dictum that «theorems are proved by those who believe in them», we are now able to discuss in the sequel three different ways for establishing the Theorem and its Corollary for arbitrary real risk-neutral drifts $\nu$.

The first of these, as discussed in Part IV, is inspired by [29], and we think Yor could have given this argument had he been aware of the financial motivation for letting the parameter $\nu$ be negative. In fact, the key idea is to try to bypass the difficulties of Bessel processes of negative index $\nu$ by Girsanov transforming to the simpler Bessel processes of index zero. This theme is developed also in the third approach discussed in Part VI. Here, the idea is not to Girsanov transform Bessel processes derived from the geometric Brownian motion driving the underlying. Instead, Girsanov transform this geometric Brownian motion itself; an idea we distilled from [27]. The effect of this is that zero drift Bessel processes enter right from the beginning, and the result is a uniform argument based on these most natural Bessel processes.

In comparison to these two approaches, our second approach discussed in Part V seems somewhat novel. The idea is to combine stochastic methods with complex analytic ones. The net effect here is that with an input of some standard result from the latter area like the identity theorem, it is not Bessel processes which now have to be dealt with but Brownian motion. For this it is moreover not required to work on the process level, as it is sufficient to work on the level of expectations. So this second approach seems to be an example of a rather promising methodology for solving problems, which is to systematically enhance stochastic techniques with complex analytic ones.

\section{Part III. Laplace transforms if $\nu \geqslant 0$}

9. Preliminaries on Bessel processes. As a preliminary to establishing the Laplace transforms of $\S 8$, this section collects a number of pertinent facts from the theory of Bessel processes. This theory is patterned after the example of the Bessel processes of integer dimension $\delta \geqslant 2$, which are defined by taking the Euclidean distance from the origin of a Brownian motion in dimension $\delta$. Applying Itô's Lemma, their infinitesimal generator $\mathscr{A}$ 
is seen to be given by

$$
\mathscr{A} f(x)=\frac{1}{2} f^{\prime \prime}(x)+\frac{2 \nu+1}{2 x} f^{\prime}(x),
$$

for any function $f$ in $C_{b}^{2}\left(\mathbf{R}_{>0}\right)$. This notion makes sense for any real number $\delta$, and the real-valued diffusion associated to $\mathscr{A}$ using the Volkonskii construction, see for instance [14, Theorem 4.3.3, p. 91], is the Bessel processes $R^{(\nu)}$ on $[0, \infty)$ with index $\nu=(\delta / 2)-1$. While Bessel processes of nonnegative indices $\nu$ stay positive if started with a positive value at time zero, Bessel processes of negative indices $\nu$ develop some pathologies. As explained in [19, XI, $\S 1]$, in this case they hit zero. If $-1<\nu<0$, they are thereupon instantantaneously reflected and never become negative. For $\nu=-1$ they continue at zero.

This matters for the second way of defining Bessel processes of arbitrary dimension $\delta$. In fact, the focus here is on squares of Bessel processes. Applying Itô's lemma, they are the continuous strong solutions of the stochastic differential equation $d \rho_{t}=2 \delta d t+2 \sqrt{\left|\rho_{t}\right|} d B_{t}, \rho_{0}=1[19, \mathrm{XI}, \S 1]$. These stochastic differential equations make sense for any real number $\delta$ and have a unique continuous strong solution also if $\delta$ is smaller than two. The so obtained processes are studied in $[30, \S 3]$. For nonnegative indices $\nu$, they coincide with the squares of the corresponding Bessel processes of index $\nu$. They develop some pathologies for negative indices $\nu$. In this case, they hit zero if started with a positive value at time zero, and if $\nu<-1$, they even continue negative. Notice that in such situations their square roots are purely imaginary, and so cannot coincide with any of the Bessel processes constructed above. However, these two ways of extending the notion of Bessel processes do coincide for processes started at time zero at a positive value up to the first time zero is hit. This essentially is the reason behind the following Lamperti identity, which may nevertheless be surprising.

Lemma. For the index $\nu$ any real number, we have the factorization:

$$
e^{B_{t}+\nu t}=R^{(\nu)}\left(A_{t}^{(\nu)}\right)
$$

for any $t>0$, where $A_{t}^{(\nu)}=\int_{0}^{t} e^{2\left(B_{w}+\nu w\right)} d w$ is Yor's process.

For $\nu \geqslant 0$ a proof is given in $[28, \S 2]$ while the general case is now contained as exercise XI (1.28), p. 452 in the third edition of [19]. We are indebted to Yor for this and for kindly supplying us with the following argument.

The idea is to apply the Itô rule to the square $Z_{t}$ of $Y_{t}=\exp \left(\nu t+B_{t}\right)$ to get

$$
Z_{t}=2(\nu+1) \int_{0}^{t} Z_{w} d w+2 \int_{0}^{t} Z_{w} d B_{w}
$$


Time change the process using the inverse function $\tau(t)=\inf \left\{u \mid \int_{0}^{u} Z_{w} d w>\right.$ $t$ t to Yor's process $A^{(\nu)}$ to get

$$
Z_{\tau(t)}=2(\nu+1) t+2 \int_{0}^{\tau(t)} Z_{w} d B_{w}
$$

To interpret the stochastic integral in this sum, apply the basic time change formalism for stochastic processes as in $[17, \S 8.5]$ to obtain

$$
\int_{0}^{\tau(t)} Z_{w} d B_{w}=\int_{0}^{t} Z_{w} \sqrt{\tau^{\prime}(w)} d W_{w}
$$

where $W_{t}$ is defined as the stochastic integral $W_{t}=\int_{0}^{\tau(t)} \sqrt{Z_{w}} d B_{w}$ and is a Brownian motion. Using the inverse function theorem of calculus, the derivative of $\tau$ is equal to the reciprocal of the derivative with respect to time of Yor's process $A^{(\nu)}$ at time $w$. Hence it is equal to the reciprocal of $Z_{w}$. On substitution we so identify the time-changed process $Z$ as a continuous solution to the stochastic differential equation for the square of the Bessel process of index $\nu$ :

$$
Z_{\tau(t)}=2(\nu+1) t+2 \int_{0}^{t} \sqrt{Z_{w}} d W_{w} .
$$

Using the uniqueness of the solution of these stochastic differential equations, the time-changed process $Z$ is the square of the Bessel process of index $\nu$. Reversing the time change, this translates into

$$
Y_{t}^{2}=\left(R_{t}^{(\nu)}\right)^{2}\left(A_{t}^{(\nu)}\right)
$$

To establish the identity of the Lemma, we have to take square roots. This is not a problem if $\nu$ is nonnegative since then the Bessel process takes nonnegative values only. It does pose a problem if $\nu$ is negative. In this case, however, recall that the Bessel process starts at time zero with a positive value. Since it is continuous by hypothesis, it will stay positive until it first hits zero at time $t^{*}>0$. Since the process $A^{(\nu)}$ starts at zero at time zero, there is a latest point in time $t^{* *}$, infinity admitted, such that $A^{(\nu)}$ is smaller than $t^{*}$ at all points in time $t$ smaller than $t^{* *}$. Thus we have the required identity at least for all points in time $t$ smaller than $t^{* *}$. Now $Y_{t}$ is never zero. Since the processes on both sides of the identity are continuous in time, $t^{* *}$ must be infinity, and the proof is complete.

10. Computing Laplace transforms if $\nu \geqslant 0$. This section is the first step in the proof of the integral representation of $\S 8$ Theorem for the Laplace transform

$$
F_{G Y, a}(z)=\int_{0}^{\infty} e^{-z x} f_{G Y, a}(x) d x=\mathscr{L}\left(f_{G Y, a}\right)(z),
$$


where, with the concepts of $\S 3$ and $\S 6$, we have $f_{G Y, a}(x)=\mathbf{E}^{\mathbf{Q}}\left[\left(A_{x}^{(\nu)}-a\right)^{+}\right]$, for any positive real numbers $a$ and $x$. We now explain why one needs to restrict the probabilistic arguments of [12] and apply them mutatis mutandis in order to arrive at the following lemma.

Lemma. The assertions of $\S 8$ Theorem are valid if $\nu=2 \sigma^{-2} \varpi-1 \geqslant 0$.

We are very indebted to Yor for correspondence and discussions about this result, and are very grateful for his kind support. In the sequel, we want to indicate the key steps of the proof following [12], while trying to incorporate his suggestions. Hopefully, no pitfalls have remained undetected.

The basic idea is to make time stochastic using the Lamperti identity

$$
e^{\nu w+B_{w}}=R^{(\nu)}\left(A_{w}^{(\nu)}\right)
$$

for all positive real numbers $w$ as has been discussed in the preceding section. Here, $R^{(\nu)}$ is the Bessel process of index $\nu$ with $R^{(\nu)}(0)=1$.

On applying this Lamperti identity, $A^{(\nu)}$ has the double role of both control variable and stochastic clock. That the «strike price» $a$ is independent of time now becomes essential. It makes possible to transcribe the condition on the control variable to be bigger than $a$ as the inverse time change

$$
\tau_{\nu, a}=\inf \left\{u \mid A_{u}^{(\nu)}>a\right\}
$$

for the stochastic clock. This is the key idea for obtaining the representation

$$
f_{G Y, a}(w)=\mathbf{E}^{\mathbf{Q}}\left[\frac{e^{2(\nu+1)\left(w-\tau_{\nu, a}\right]^{+}}-1}{2(\nu+1)}\left(R_{a}^{(\nu)}\right)^{2}\right],
$$

for all $w>0$. Indeed, fix any positive real number $x$, and consider the process $A^{(\nu)}$ at $x$ on the set of all events, where $\tau_{\nu, a}$ takes values less than or equal to $x$. Break the integral defining $A^{(\nu)}(x)$ at $\tau_{\nu, a}$. The first summand then is $A^{(\nu)}$ at time $\tau_{\nu, a}$ and so is equal to $a$. In the second summand, restart the Brownian motion in the exponent of the integrand at $\tau_{\nu, a}$ shifting the variable of integration accordingly. The second integral then is the product of $\exp \left(2\left(B\left(\tau_{\nu, a}\right)+\nu \tau_{\nu, a}\right)\right)$ times $A^{(\nu)}$ at $x-\tau_{\nu, a}$, by abuse of language after having applied the Strong Markov property. This last process is such that it is independent of the information at time $\tau_{\nu, a}$. Unravelling the definition of $\tau_{\nu, a}$, the first above factor so is the square of the Bessel process $R^{(\nu)}$ at time $a$. Now taking the expectation conditional on the information at $\tau_{\nu, a}$, we thus get:

$$
\mathbf{E}^{\mathbf{Q}}\left[\left(A_{x}^{(\nu)}-a\right)^{+} \mid \mathscr{F}_{\tau_{\nu, a}}\right]=\left(R_{a}^{(\nu)}\right)^{2} \mathbf{E}^{\mathbf{Q}}\left[A_{\left[x-\tau_{\nu, a}\right]^{+}}^{(\nu)}\right] .
$$

On substitution for the expectation of $A^{(\nu)}(w)$ from $\S 4$ Lemma or using [29, $\S 4]$, the required expression for $f_{G Y, a}$ follows. 
At first sight this appears to complicate the problem. However, it is just what is especially suited to the Laplace transform $F_{G Y, a}$ of $f_{G Y, a}$ now given by:

$$
F_{G Y, a}(z)=\int_{0}^{\infty} e^{-z w} \mathbf{E}^{\mathbf{Q}}\left[\frac{e^{2(\nu+1)\left[w-\tau_{\nu, a}\right]^{+}}-1}{2(\nu+1)}\left(R_{a}^{(\nu)}\right)^{2}\right] d w .
$$

Still, for computing this integral one wants to interchange the Laplace integral with the expectation $\mathbf{E}^{\mathbf{Q}}$. If $z$ is real, it seems best to follow Yor's proposal for justifying this. Indeed, with the integrand of the double integral in question positive and measurable, apply Tonelli's theorem now but justify only in a later step that any of the resulting integrals are finite. The case of a general argument $z$ is reduced to this case by considering the absolute value of the integrand, and the result is the identity

$$
F_{G Y, a}(z)=\frac{1}{z(z-2(\nu+1))} \mathbf{E}^{\mathbf{Q}}\left[e^{-z \tau_{\nu, a}}\left(R^{(\nu)}\right)^{2}\right]
$$

of measurable functions for any complex number $z$ with $\operatorname{Re}(z)>2(\nu+1)$. The idea for identifying the expectation in the numerator as $D_{\nu}(a, z)$ then is to condition on the Bessel process to obtain

$$
D_{\nu}(a, z)=\int_{0}^{\infty} x^{2} \mathbf{E}^{\mathbf{Q}}\left[e^{-z \tau_{\nu, a}} \mid R_{a}^{(\nu)}=x\right] p_{\nu, a}(1, x) d x,
$$

where $p_{\nu, a}$ is the time- $a$ semigroup density of the Bessel process of index $\nu$ starting at 1 at time zero. Following [12, p. 362] we make this integral explicit by making the single factors of its integrand explicit. For this, work with the results recalled in $[12, \S 2]$. With respect to the conditional expectation factor, under the hypothesis $\nu \geqslant 0$, Yor has computed it at positive real arguments $z$ in [27, Théorème 4.7, p. 80] (see also [12, Lemma 2.1 and Proposition 2.6]). Using analytic continuation, the validity of his result can be seen to extend to the arguments $z$ in the right half-plane required in the present situation. This then gives for the conditional expectation factor in $D_{\nu}(a, z)$ the following expression as a quotient of $I$-Bessel functions:

$$
\mathbf{E}^{\mathbf{Q}}\left[e^{-z \tau_{\nu, a}} \mid R^{(\nu)}(a)=w\right]=\frac{I_{\sqrt{2 z+\nu^{2}}}}{I_{\nu}}\left(\frac{w}{a}\right) .
$$

Explicit expressions for the Bessel semigroups $p_{\nu, a}$ have been known for $\nu>-1$ for some time, see [27, (4.3), p. 78] or [12, Proposition 2.2]. The density $p_{\nu, a}(1, w)$ of the time- $a$ Bessel semigroup with index $\nu$ and starting point 1 is

$$
p_{\nu, a}(1, w)=\frac{w^{\nu+1}}{a} e^{-\left(1+w^{2}\right) /(2 a)} I_{\nu}\left(\frac{w}{a}\right) .
$$

Nothing seemed to have been known about such densities if $\nu \leqslant-1$ before [30]. However, the results proved there for $\nu<-1$ still need to be 
handled with care as it will be explained in $\S 14$. The upshot is that, in accordance with $[12, \S 2]$, the above decomposition of $D_{\nu}(a, z)$ seems to give explicit results without further qualifications only if $\nu \geqslant 0$. Then, however, we have the required result

$$
D_{\nu}(a, z)=\frac{e^{-1 /(2 a)}}{a} \int_{0}^{\infty} e^{-x^{2} /(2 a)} x^{\nu+3} I_{\sqrt{2 z+\nu^{2}}}\left(\frac{x}{a}\right) d x .
$$

There is a further technical point to be taken care of herein: choose the principal branch of the logarithm to define the square root on the complex plane with the nonpositive real line deleted.

To complete the Tonelli argument proposed to us by Yor and to complete the proof, we have to establish the finiteness of this integral for any fixed complex number $z$ with $\operatorname{Re}(z)>2(\nu+1)$. This is a consequence of $\S 11$ Proposition's convergence analysis of these integrals, and granting this result, the proof of the Lemma is complete.

11. Integrability analysis. In terms of the concepts of $\S 3$, this section studies finiteness of the integrals

$$
D_{\nu}(a, z)=\frac{e^{-1 /(2 a)}}{a} \int_{0}^{\infty} e^{-x^{2} /(2 a)} x^{\nu+3} I_{\sqrt{2 z+\nu^{2}}}\left(\frac{x}{a}\right) d x
$$

for any real $a>0$ in terms of their complex parameters $\nu$ and $z$. The precise result is the following proposition.

Proposition. Let $\varepsilon \geqslant 0$ be any real number. If $|\operatorname{Im}(\nu)| \leqslant \varepsilon$, the integrals $D_{\nu}(a, z)$ are finite for any complex $z$ with real part $\operatorname{Re}(z)>2 \varepsilon^{2}$.

The Proposition depends on the following result about the complex square root associated to the principal branch of the complex logarithm.

Lemma. Let $\varepsilon \geqslant 0$ be any real number. For any complex $\nu$ with $|\operatorname{Im}(\nu)| \leqslant \varepsilon$ we then have

$$
\operatorname{Re}\left(\sqrt{2 z+\nu^{2}}\right)>|\operatorname{Re}(\nu)|
$$

for any complex $z$ with $\operatorname{Re}(z)>2 \varepsilon^{2}$.

A proof of the Lemma based on a close analysis of the square root can be found in $[2, \S 10]$. To prove the Proposition, finiteness of $D_{\nu}(a, z)$ under the conditions of the Proposition follows by combining the above square root lemma with the asymptotic behaviour of the Bessel function factor of its integrand near the origin and towards infinity. Indeed, setting $\mu=\sqrt{2 z+\nu^{2}}$, from $[15, \S 5.11]$ recall that $I_{\mu}$ is a continuous function on the positive real line in particular whose asymptotic behaviour for large real arguments is

$$
I_{\mu}(\xi) \approx \frac{e^{\xi}}{\sqrt{2 \pi \xi}} \quad \text { for } \quad \xi \rightarrow \infty .
$$


Hence the factor $\exp \left(-x^{2} /(2 a)\right)$ dominates the asymptotic behaviour of the integrand of $D_{\nu}(a, z)$ with $x$ to infinity, whence its integrability away from the origin. On the other hand, from $[15, \S 5.7]$ we have for real arguments near zero

$$
I_{\mu}(\xi) \approx \frac{\xi^{\mu}}{2^{\mu} \Gamma(1+\mu)} \quad \text { for } \quad \xi \downarrow 0 .
$$

Thus, if the real part of $\mu+\nu+4$ is positive, or equivalently, if we have

$$
\operatorname{Re}(\mu)>-(\operatorname{Re}(\nu)+4),
$$

no integrability problems arise for $x$ near the origin. Under the conditions of the Proposition, on the other hand, the above Lemma gives $\operatorname{Re}(\mu)>|\operatorname{Re}(\nu)|$. Since this last inequality implies the former, the proof of the Proposition is complete.

\section{Part IV. Laplace transforms in the general case: using Girsanov transforms of Bessel processes}

12. Further preliminaries on Bessel processes. Our first way of extending the results of $\S 10$ to negative indices $\nu$ requires further preliminaries on Bessel processes from $[29, \S 2]$. Recall that Bessel processes where the index $\nu$ is any real number are the real-valued diffusions whose infinitesimal generators $\mathscr{A}$ are given by

$$
\mathscr{A} f(x)=\frac{1}{2} f^{\prime \prime}(x)+\frac{2 \nu+1}{2 x} f^{\prime}(x)
$$

for any function $f$ in $C_{b}^{2}\left(\mathbf{R}_{>0}\right)$. To describe the law $P_{\mu, u}$ on $C\left(\mathbf{R}_{\geqslant 0}, \mathbf{R}_{\geqslant 0}\right)$ of $R^{(\nu)}$ if this process starts at the nonnegative real $u$, let $\rho$ be the canonical process on $C\left(\mathbf{R}_{\geqslant 0}, \mathbf{R}_{\geqslant 0}\right)$; recall it operates as evaluation map: $\rho_{a}(f)=f(a)$. If $\mathscr{R}$ is the canonical filtration with $\mathscr{R}_{a}$ equal to the sigma algebra generated by the $\rho_{s}$ with $s \leqslant a$, we then have the mutual absolute continuity relation:

Lemma. If the Bessel process of any real index $\nu$ is started at any nonnegative real $u$, its law is related to that of the zero index Bessel process started at $u$ as follows

$$
P_{\mu, u \mid \mathscr{R}_{a} \cap\left\{a<T_{0}\right\}}=\left(\frac{\rho_{a}}{u}\right)^{\mu} \exp \left(-\frac{\mu^{2}}{2} \int_{0}^{a} \frac{d s}{\rho_{s}^{2}}\right) P_{0, u \mid \mathscr{R}_{a}}
$$

where $T_{0}$ is the first passage time of $\rho$ to zero.

This is proved as an application of Girsanov's theorem by exchanging drifts in the stochastic differential equation of $\S 9$, and has the following

Corollary. For any complex $z$ with positive real part, and any nonnegative real $r$,

$$
\mathbf{E}_{u}^{0}\left[\exp \left(-z \int_{0}^{a} \frac{d s}{\rho_{s}^{2}}\right) \mid \rho_{a}=r\right]=\frac{I_{\sqrt{2 z}}}{I_{0}}\left(\frac{u r}{a}\right),
$$

where the expectation is taken with respect to the law $P_{0, u}$. 
The Corollary is proved in two steps. If $z$ is any nonnegative real, $T_{0}=\infty$, and we have the explicit expressions for the densities $p_{\mu, a}(u, r)$ of the of the Bessel semigroups already encountered in $\S 10$ and related to the law via $P_{\mu, u}(a, d r)=p_{\mu, a}(u, r) d r$ :

$$
p_{\mu, a}(u, r)=\left(\frac{r}{u}\right)^{\mu} \frac{r}{a} \exp \left(-\frac{1}{2 a}\left(u^{2}+r^{2}\right)\right) I_{\mu}\left(\frac{u r}{a}\right),
$$

for any nonnegative reals $u, a>0$ and $r$. And so the Corollary follows on taking expectations in the absolute continuity relation of the Lemma. Observing that both sides of the identity to be proved are analytic functions in $z$ on the right half-plane, the general case then follows by analytic continuation as a second step.

13. First proof of the Laplace transform using Bessel processes. Resuming the discussion of $\S 10$, we are now able to complete the proof of $\S 8$ Theorem in the way it might have been envisaged by Yor: based on a careful analysis of Bessel processes. Recall that we still need explicitly compute for negative normalized risk-neutral drifts $\nu$, the risk-neutral expectations

$$
\mathbf{E}^{\mathbf{Q}}\left[e^{-z \tau_{\nu, a}}\left(R_{a}^{(\nu)}\right)^{2}\right]
$$

where $a>0$ and $\operatorname{Re}(z)$ is positive and sufficiently big in particular. From the time change part of the argument in $\S 9$, recall that $\tau_{\nu, a}$ as the inverse time change of the process $A^{(\nu)}$ at time $a$ is given by $\tau_{\nu, a}=\int_{0}^{a} d s /\left(R_{s}^{(\nu)}\right)^{2}$. Again using $\S 9$ 's Lamperti relation

$$
e^{B_{w}+\nu w}=R^{(\nu)}\left(A_{w}^{(\nu)}\right)
$$

which is valid for $w \geqslant 0$, the point now is that $a$ is smaller than the first passage time to zero $T_{0}$ of the Bessel process $R^{(\nu)}$. Put differently, this Bessel process which starts in 1 at time zero still is positive at time $a$. Using the absolute continuity relation of $\S 12$ Lemma,

$$
\mathbf{E}^{\mathbf{Q}}\left[\left(R_{a}^{(\nu)}\right)^{2} e^{-z \tau_{\nu, a}}\right]=\mathbf{E}_{1}^{0}\left[\rho_{a}^{2} \exp \left\{-z \int_{0}^{a} \frac{d s}{\rho_{s}^{2}}\right\} \rho_{a}^{\nu} \exp \left\{-\frac{\nu^{2}}{2} \int_{0}^{a} \frac{d s}{\rho_{s}^{2}}\right\}\right] .
$$

Conditioning on the index- 0 Bessel process thus gives

$$
\begin{aligned}
& \mathbf{E}^{\mathbf{Q}}\left[e^{-z \tau_{\nu, a}}\left(R_{a}^{(\nu)}\right)^{2}\right] \\
& \quad=\int_{0}^{\infty} \mathbf{E}_{1}^{0}\left[\exp \left\{-\left(z+\frac{\nu^{2}}{2}\right) \int_{0}^{a} \frac{d s}{\rho_{s}^{2}}\right\} \mid \rho_{a}=\rho\right] \rho^{\nu+2} p_{0, a}(1, \rho) d \rho .
\end{aligned}
$$

With the explicit form of the semigroup recalled in $\S 12$, applying $\S 12$ Corollary gives

$$
\mathbf{E}^{\mathbf{Q}}\left[e^{-z \tau_{\nu, a}}\left(R_{a}^{(\nu)}\right)^{2}\right]=\frac{1}{a} e^{-1 /(2 a)} \int_{0}^{\infty} I_{\sqrt{2 z+\nu^{2}}}\left(\frac{\rho}{a}\right) \rho^{\nu+3} e^{-\rho^{2} /(2 a)} d \rho
$$


as desired. An application of $\S 11$ Proposition then shows finiteness of this integral if $z_{0}=\operatorname{Re}(z)$ is positive and bigger than $2(\nu+1)$, and the first proof of $\S 8$ Theorem is complete. Note that meanwhile the argument of [32, p. 97-99] seems to corroborate our statement that this proof is very much in the spirit of Yor.

14. Vista on the use of Bessel processes. The strategy of the preceding argument is to bypass the difficulties brought about by Bessel processes with negative indices by Girsanov transforming to Bessel processes of index zero. One could ask about a direct attack in the spirit of $\S 10$. This would require possession of explicit expressions for both the densities of the respective Bessel semigroups and the pertinent conditional expectations of $\exp \left(-z \tau_{\nu, a}\right)$. The recent work of Göing-Jaeschke and Yor in [30] now in particular provides certain analytic expressions for the densities. However, it is not Bessel processes as considered in $\S 12$ which are studied there, but rather processes obtained as strong solutions to the stochastic differential equations of squared Bessel processes, as mentioned in $\S 9$. Recall that for negative indices, the latter processes become negative and their square roots, which should give the Bessel processes, then are purely imaginary. Still, the two notions of Bessel processes thus obtained coincide on their respective positive range. There, we have Bessel semigroup densities based on those derived in $[30, \S 3$ Proposition 2, p. 21]. For indices $\nu<-1$ and time- 0 starting values $y>0$, they are given by

$p_{\nu, t}(x, y)=h(x, y, \delta, t) \exp \left(\frac{y-x}{2 t}\right) \int_{0}^{1}(1-w)^{\frac{2(\mu-1)}{w^{\mu}}} \exp \left(\frac{1}{2 t}\left(x w-\frac{y}{w}\right)\right) d w$ defining

$$
h(x, y, \delta, t)=\frac{1}{\Gamma^{2}(\mu-1)}(x y)^{(\mu-1) /\left[2^{2-\delta}(2-\delta)\right]} t^{\delta-3}
$$

and with $\delta=2(1+\nu)<0$ and $\mu=1-\nu$. However, these densities are in terms of new classes of special functions. The clarification of their relations to those for Bessel processes of nonnegative indices is but one of the problems that require further study.

\section{Part V. Laplace transforms in the general case: combining stochastics and complex analysis}

15. Remarks on general philosophy. The discussion up to now has focused in particular on extension at the process level. The actual valuation problem, however, is not at the process level but at the expectation level. From this point of view, $\S 10$ has identified two functions $f$ and $g$ in the variable $\nu$ and has proved them to be equal for $\nu$ nonnegative. One would like to have this equality also for negative $\nu$, and thus extend the validity of the identity $f=g$ from the nonnegative real line to the whole real line. Such 
situations quite commonly appear in problems in analysis and are addressed there using analytic continuation. However, functions become amenable to complex analytic methods only on open subsets of the complex plane. Thus the identity theorem of complex analysis asserts that two functions on a connected open subset of the complex plane are equal if they are analytic and agree on a convergent sequence there only. So it is in fact no longer possible to stick to real numbers only. As a subset of the complex plane they are closed with an empty interior. At this stage, however, nothing is known about the functions of $\S 10$ if $\nu$ is outside the nonnegative real line. In particular, it is not known if they exist at all, and this needs to be established together with their analyticity properties. Of these two functions, $D_{\nu}(a, z)$ is already given as an explicit analytic expression, while the other function is not. In fact, it is not explicit at all, as it is defined as the Laplace transform of a certain expectation. The question to be tackled here is then how to get explicit analyticity properties from such nonexplicit stochastic concepts. In the present situation, we attack this in two stages. First, establish analyticity properties of the expectation. Then, in a second step, study how these are preserved on taking Laplace transforms. As it turns out, using standard results from complex analysis, we find that it is not Bessel precesses which enter into the analysis but simply Brownian motion. All of this may be regarded as an instance for why enhancing stochastics by complex analytic methods seems quite an interesting and promising line of thought.

16. First step - analyticity of the function $D_{\nu}(a, z)$. As a first step in the analytic continuation argument, this section studies the analytic properties of the generalized first Weber integral $D_{\nu}(a, z)$ of $\S 8$ Theorem. On choosing the square root associated to the principal branch of the logarithm recall

$$
D_{\nu}(a, z)=\frac{e^{-1 /(2 a)}}{a} \int_{0}^{\infty} e^{-x^{2} / /(2 a)} x^{\nu+3} I_{\sqrt{2 z+\nu^{2}}}\left(\frac{x}{a}\right) d x
$$

is finite for any positive real number $a$ and for any complex numbers $z$ and $\nu$ such that the real part of $\nu+4+\left(2 z+\nu^{2}\right)^{1 / 2}$ is positive as a consequence of $\S 11$ Proposition's integrability analysis. Using the confluent hypergeometric function $\Phi$ discussed in $[15, \S 9.9]$, the precise analyticity result to be proved is the following

Proposition. Let $a$ be any positive real and $\varepsilon$ any nonnegative real. For any complex number $\nu$ with $|\operatorname{Im}(\nu)| \leqslant \varepsilon$ we then have

$$
\begin{aligned}
D_{\nu}(a, z)= & \Gamma\left(\frac{\nu+4+\mu}{2}\right) \frac{1}{\Gamma(\mu+1)} \\
& \times \Phi\left(\frac{\nu+4+\mu}{2}, \mu+1 ; \frac{1}{2 a}\right) e^{-1 /(2 a)}(2 a)^{(\nu+2-\mu) / 2}
\end{aligned}
$$

if $z$ is any complex with $\operatorname{Re}(z)>2 \varepsilon^{2}$ setting $\mu=\sqrt{2 z+\nu^{2}}$. 
Corollary. Let $a$ be any positive real and $\varepsilon$ any nonnegative real. For any complex number $z$ with $\operatorname{Re}(z)>2 \varepsilon^{2}$, sending $\nu$ to $D_{\nu}(a, z)$ then defines an analytic map on the set of all complex numbers $\nu$ with $|\operatorname{Im}(\nu)| \leqslant \varepsilon$.

Both results are based on $\S 11$ Proposition which gives finiteness of $D_{\nu}(a, z)$ under their conditions on $\nu, a$, and $z$. Combining this with the analyticity properties of $\Phi$ discussed in $[15, \S 9.9]$ and those of the gamma function, the Corollary follows from the Proposition. The proof of the Proposition then reduces to explicitly computing $D_{\nu}(a, z)$. For this we modify the quite typical discussion in $[25, \S 13.3$, p. $393 \mathrm{f}]$ of Hankel's generalization of Weber's first integral. The idea is to expand the modified Bessel function in the integrand of

$$
I=\int_{0}^{\infty} e^{-x^{2} /(2 a)} x^{\nu+3} I_{\mu}\left(\frac{x}{a}\right) d x
$$

into its series of $[15, \S 5.7]$ and integrate term by term. Using $[15, \S 9.9]$ this is justified by the absolute convergence of the series for the confluent hypergeometric series which is to result, and we get

$$
I=\frac{1}{(2 a)^{\mu}} \sum_{n=0}^{\infty} \frac{1}{\Gamma(\mu+1+n)} \frac{(2 a)^{-2 n}}{n !} \int_{0}^{\infty} e^{-x^{2} /(2 a)} x^{\nu+3+\mu+2 n} d x .
$$

Changing variables $y=(2 a)^{-1} x^{2}$, compute any $n$th integral as

$$
\int_{0}^{\infty} e^{-x^{2} /(2 a)} x^{\nu+3+\mu+2 n} d x=\frac{1}{2} \Gamma\left(\frac{\nu+\mu+4}{2}+n\right)(2 a)^{(\nu+\mu+4) / 2+n} .
$$

Extracting the series of the pertinent confluent hypergeometric function we thus get

$$
I=\frac{1}{2} \frac{\Gamma((\nu+4+\mu) / 2)}{\Gamma(\mu+1)} \Phi\left(\frac{\nu+4+\mu}{2}, \mu+1 ; \frac{1}{2 a}\right)(2 a)^{(\nu-\mu+4) / 2} .
$$

Multiplying this expression with $\exp \left(-(2 a)^{-1}\right) / a$, the Proposition follows.

17. Second step - analyticity of the functions $f_{G Y, a}$. Establishing analyticity results in $\nu$ about the Laplace transform of the expectation defining non-Asian option prices combines insights from stochastics with insights of an analytic nature. We establish this result in two steps. As a first step, in this section any of the auxiliary functions $f_{G Y, a}$ of $\S 7$ is considered as function in the variable $\nu$

$$
L(x, \nu)=\mathbf{E}^{\mathbf{Q}}\left[\left(A_{x}^{(\nu)}-a\right)^{+}\right]
$$

for any fixed positive real numbers $a$ and $x$. Using $\S 10$ Lemma, we know it is defined for nonnegative real numbers $\nu$. However, this has been achieved in a very indirect way only: for these values of $\nu$ the Laplace transforms of the corresponding functions $f_{G Y, a}$ have been shown to be finite. Now more is true indeed: 
Lemma. For any $x>0$, the function $\nu \mapsto L(x, \nu)$ extends to a function on the complex plane which is analytic at each point, and for which we have the majorization

$$
|L(x, \nu)| \leqslant e^{x \operatorname{Im}^{2}(\nu) / 2} \mathbf{E}^{\mathbf{Q}}\left[A_{x}^{(\operatorname{Re}(\nu))}\right] .
$$

For the proof of the Lemma now set $f(w)=(w-a)^{+}$. Applying Girsanov's theorem such that $W_{x}=\nu x+B_{x}$ becomes a standard Brownian motion, and dropping reference to this new measure, we get

$$
L(x, \nu)=\mathbf{E}\left[f\left(A_{x}^{(0)}\right) e^{\nu W_{x}}\right] e^{-x \nu^{2} / 2} .
$$

For establishing the analyticity statement of the Lemma, it is thus sufficient to show that the expectation factor is analytic in any complex number $\nu$. This is true by definition if we have the convergent series

$$
\mathbf{E}\left[f\left(A_{x}^{(0)}\right) e^{\nu W_{x}}\right]=\sum_{m=0}^{\infty} \frac{\nu^{m}}{m !} \mathbf{E}\left[f\left(A_{x}^{(0)}\right) W_{x}^{m}\right]
$$

for all $\nu$. For this it is sufficient to show that the series is absolutely convergent for all $\nu$. Using the Cauchy-Schwarz inequality this is implied by the convergence of

$$
\sum_{m=0}^{\infty} \frac{|\nu|^{m}}{m !} \sqrt{\mathbf{E}\left[f^{2}\left(A_{x}^{(0)}\right)\right]} \sqrt{\mathbf{E}\left[W_{x}^{2 m}\right]}
$$

for all $\nu$. Herein $\mathbf{E}\left[f^{2}\left(A_{x}^{(0)}\right)\right]$ is majorized by the second moment of Yor's zero drift process $A^{(0)}$ at $x$, and so is finite from [29, $\left.\S 4\right]$. Since the factors $\mathbf{E}\left[W_{x}^{2 m}\right]$ are majorized by $\pi^{-1 / 2}(2 x)^{m} m$ ! for all $m \geqslant 0$, convergence follows using the ratio test. Actually, we have so established yet another upper bound to the price of the Asian option.

To establish the majorization of the Lemma, taking absolute values inside the expectation in the above Girsanov representation of $L(x, \nu)$ gives:

$$
|L(x, \nu)| \leqslant \mathbf{E}\left[f\left(A_{x}^{(0)}\right)\left|e^{\nu W_{x}}\right|\right]\left|e^{-x \nu^{2} / 2}\right| .
$$

The absolute value of the exponential factors are the exponentials of the real parts of the respective arguments. Majorizing the function in Yor's zero drift process by this process itself, we get

$$
|L(x, \nu)| \leqslant e^{x \operatorname{Im}^{2}(\nu) / 2} \mathbf{E}\left[A_{x}^{(0)} e^{\operatorname{Re}(\nu) W_{x}}\right] e^{-x \operatorname{Re}^{2}(\nu) / 2} .
$$

Reversing the Girsanov transformation then completes the proof of the Lemma. 
18. Third step - analyticity of the transforms $F_{G Y, a}$. While the preceding section has studied the expectations

$$
L(x, \nu)=\mathbf{E}^{\mathbf{Q}}\left[\left(A_{x}^{(\nu)}-a\right)^{\dagger}\right]
$$

for any fixed positive real numbers $a$ and $x$ as function in the complex variable $\nu$ only, this section moreover treats $x$ as a variable. If $\nu$ is any nonnegative real number, we have from $\S 10$ Lemma that the integrals of the Laplace transforms

$$
F(\nu)(z)=\int_{0}^{\infty} e^{-z x} L(x, \nu) d x
$$

are finite if $\operatorname{Re}(z)>2(\nu+1)$. In this section we give an independent proof of the following more general statement.

Proposition. For any complex number $z$ with a positive real part, the map sending $\nu$ to $F(\nu)(z)$ is analytic in all complex numbers $\nu$ with real parts $\operatorname{Re}(z)>\frac{1}{2} \operatorname{Im}^{2}(\nu)+2(\operatorname{Re}(\nu)+1)$.

The proof of the Proposition is based on the following lemma.

Lemma. For any complex $\nu$, the Laplace transform $F(\nu)(z)$ is finite for any complex $z$ with $\operatorname{Re}(z)>\max \left\{0, \frac{1}{2} \operatorname{Im}^{2}(\nu)+2(\operatorname{Re}(\nu)+1)\right\}$.

$\mathrm{Pr}$ o of of $\mathrm{th}$ e $\mathrm{L}$ e $\mathrm{m} \mathrm{m}$ a. As first step in proving the Lemma, we establish for any complex number $\nu$ the majorization

$$
|F(\nu)(z)| \leqslant \int_{0}^{\infty} \exp \left\{-\left(\operatorname{Re}(z)-\frac{1}{2} \operatorname{Im}^{2}(\nu)\right) x\right\} \mathbf{E}^{\mathbf{Q}}\left[A_{x}^{(\operatorname{Re}(\nu))}\right] d x
$$

for any complex number $z$ in the sense of measurable functions. Indeed, majorize the absolute value of $F(\nu)(z)$ by taking the absolute value inside the defining integral. The absolute value of the exponential factor then is equal to $\exp (-\operatorname{Re}(z) x)$. Majorizing the absolute value of $L(x, \nu)$ using $\S 17$ Lemma, the estimate follows.

Setting $\nu_{0}=\operatorname{Re}(\nu)$, now let $\operatorname{Re}(z)$ be positive and such that $\xi_{0}=$ $\operatorname{Re}(z)-\operatorname{Im}^{2}(\nu) / 2$ is bigger than $2\left(\nu_{0}+1\right)$. We compute the Laplace transform of the right-hand side of the above inequality using $\S 4$ Lemma. If $\nu_{0}$ is different from minus one,

$$
\int_{0}^{\infty} e^{-\xi_{0} x} \mathbf{E}^{\mathbf{Q}}\left[A_{x}^{\left(\nu_{0}\right)}\right] d x=\frac{1}{\xi_{0}\left(\xi_{0}-2\left(\nu_{0}+1\right)\right)},
$$

using that $\xi_{0}$ is bigger than $2\left(\nu_{0}+1\right)$ to compute the improper integrals. It converges to $\xi_{0}^{-2}$ with $\nu_{0}$ going to minus one. Thus it is seen to coincide with the Laplace transform for the case $\nu_{0}=-1$.

The Laplace transforms are finite if $\xi_{0}$ is positive and bigger than $2\left(\nu_{0}+1\right)$, and then $F(\nu)(z)$ is finite a forteriori. The proof of the Lemma is complete. 
$\mathrm{Pr}$ o of of the Prop osition. For proving the Proposition; fix any complex $z$ with a positive real part and then choose any complex $\nu_{0}$ satisfying the resulting inequality of the Proposition. In fact, the validity of this inequality then extends to all $\nu$ in a compact neighbourhood $V$ of $\nu_{0}$. As a consequence of $\S 17$ Lemma, $f_{z}(x, \nu)=\exp (-z x) L(x, \nu)$ is for any $x>0$ analytic in $\nu$ on $V$ in particular. Since $V$ is compact, the argument of the Lemma moreover shows that its absolute value is majorized by an integrable function $g$ on the real line. The function $\nu \mapsto F(\nu)(z)$ so is continuous on $V$ being obtained by integration of $f_{z}$ over the positive real line. To show it is analytic on the interior of $V$ we want to apply Morera's theorem, see [18, 10.17 , p. 208], and have to show

$$
\int_{\partial \Delta} F(\nu)(z) d \nu=0
$$

for any triangle $\Delta$ contained in the interior of $V$. Indeed, since we have shown $f_{z}$ to be integrable, applying Fubini's theorem gives

$$
\int_{\partial \Delta} F(\nu)(z) d \nu=\int_{0}^{\infty} \int_{\partial \Delta} f_{z}(x, \nu) d \nu d x .
$$

Recalling that $\nu \mapsto f_{z}(x, \nu)$ is analytic from $\S 17$ Lemma, the inner integral herein is zero by Cauchy's theorem for $\Delta$, see $[18,10.13$, p. 205]. Thus the whole double integral is zero, as was to be shown. This completes the proof of the Proposition.

19. Final step - second proof of the Laplace transform using analytic extension. It remains to pull things together and show how the results of this Part V give a second proof of the two results of $\S 8$ by using analytic extension.

First notice that $\S 8$ Corollary is implied by $\S 8$ Theorem using the computation of $D_{\nu}(a, z)$ in $\S 16$ Proposition. Thus we are reduced to prove $\S 8$ Theorem.

The proof of $\S 8$ Theorem is by analytic continuation using the identity theorem, see [18, Corollary to 10.18, p. 209]. As a consequence of $\S 10$ Lemma it remains to establish the identity in $\S 8$ Theorem for negative indices $\nu$ only. It will turn out that the existence of the Laplace transform on all complex numbers $z$ with positive real part required in the Theorem then has essentially been proved in $\S 18$ Proposition.

For establishing the crucial Laplace transform identity of $\S 8$ Theorem, thus first let $z$ be any complex number with real part $\operatorname{Re}(z)>4$ and choose $0<\varepsilon<1$. Using $\S 16$ Corollary, the generalized first Weber integral

$$
D_{\nu}(a, z)=\frac{e^{-1 /(2 a)}}{a} \int_{0}^{\infty} e^{-x^{2} /(2 a)} x^{\nu+3} I_{\sqrt{2 z+\nu^{2}}}\left(\frac{x}{a}\right) d x
$$


is analytic in $\nu$ on the $\varepsilon$ thickened real line $A_{\varepsilon}$ which consists of all complex numbers $\nu$ with $|\operatorname{Im}(\nu)|<\varepsilon$. Picture $A_{\varepsilon}$ as the band of height $2 \varepsilon$ symmetric with respect to the real axis. If we choose $\varepsilon$ so small that $\operatorname{Re}(z)>2(2+2 \varepsilon)$, we claim to have analyticity of the Laplace transform

$$
F(\nu)(z)=\int_{0}^{\infty} e^{-z x} L(x, \nu) d x
$$

as a function in $\nu$ on the $\varepsilon$ thickened half-line $B_{\varepsilon}$, that subset of $A_{\varepsilon}$ which consists of all complex numbers $\nu$ with $|\operatorname{Im}(\nu)|<\varepsilon$ and $\operatorname{Re}(\nu)<\varepsilon$. Indeed, if $\operatorname{Re}(\nu)<\varepsilon$, we have $2(2+2 \varepsilon)>2 \varepsilon+2(\operatorname{Re}(\nu)+2)$. If $|\operatorname{Im}(\nu)|<\varepsilon$, we have $2 \varepsilon>\operatorname{Im}^{2}(\nu) / 2$ since $\varepsilon<1$. Any $\nu$ in $B_{\varepsilon}$ so satisfies the inequality $\operatorname{Re}(z)>\operatorname{Im}^{2}(\nu) / 2+2(\operatorname{Re}(z)+2)$ of $\S 18$ Proposition and the claim follows.

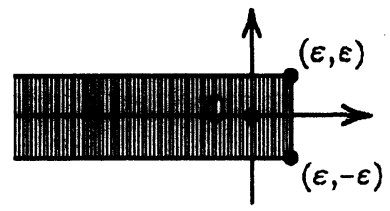

Figure 1 . The $\varepsilon$-thickened half-line $B_{\varepsilon}$.

So the functions $F(\nu)(z)$ and

$$
D_{\nu}^{*}(a, z)=\frac{D_{\nu}(a, z)}{z(z-2(\nu+1))}
$$

are analytic as functions in $\nu$ on the $\varepsilon$-thickened half-line $B_{\varepsilon}$. It is now a consequence of $\S 10$ Lemma that we have

$$
F(\nu)(z)=D_{\nu}^{*}(a, z) \quad \text { for all } \quad \nu \geqslant 0
$$

such that $2(\nu+1)<\operatorname{Re}(z)$. With $\operatorname{Re}(z)>4$ this so holds a forteriori for all $\nu \geqslant 0$ such that $2(\nu+1)<4$, i.e., for all nonnegative real numbers $\nu$ smaller than 1 . With $\varepsilon$ smaller than 1 we so have $F(\nu)(z)=D_{\nu}^{*}(a, z)$ for any $\nu$ in the subinterval $(0, \varepsilon)$ of $B_{\varepsilon}$. With $B_{\varepsilon}$ open and connected the identity theorem literally applies to give that $F(\nu)(z)=D_{\nu}^{*}(a, z)$ for all $\nu$ in $B_{\varepsilon}$. This identity then holds a forteriori for all real numbers $\nu$ in $B_{\varepsilon}$, i.e., for all $\nu<\varepsilon$. This gives $\S 8$ Theorem for any $z$ with $\operatorname{Re}(z)>4$.

To lift this last restriction on $\operatorname{Re}(z)$, notice that $\S 18$ Lemma implies $F(\nu)$ for any fixed real $\nu$ to be analytic on the half-plane $\{\operatorname{Re}(z)>2(\nu+1)\}$; this is seen by using a Morera type argument as for proving $\S 18$ Proposition. On the other hand, applying $\S 16$ Corollary, $D_{\nu}^{*}(a, z)$ as a function of $z$ is analytic on the intersection of this last half-plane with the right half-plane. The validity of the identity $F(\nu)(z)=D_{\nu}^{*}(a, z)$ thus can be analytically continued from complex numbers $z$ with $\operatorname{Re}(z)>4$ to complex numbers $z$ with $\operatorname{Re}(z)$ positive and bigger than $2(\nu+1)$, and the second proof of $\S 8$ Theorem is complete. 


\section{Part VI. Laplace transforms in the general case: a uniform proof}

20. Remarks on general philosophy. The question may arise if there is not a uniform way for computing the Laplace transforms in $\S 8$ Theorem not requiring a two step procedure. The Lamperti factorization

$$
e^{B_{w}+\nu w}=R^{(\nu)}\left(A^{(\nu)}(w)\right)
$$

of $\S 9$ Lemma may in fact offer a clue. The idea of the two arguments discussed up to now is to focus on the Bessel process side of this identity. It is the problems with Bessel processes that transcribe into their two-step approach. A possible remedy so would be to try to bring in Bessel processes not at the earliest stage but as late as possible. The Lamperti factorization suggests to focus on the geometric Brownian motion of its left-hand side. While Yor has already made extensive use of this device, we explain in the sequel what seems to be a most natural adaption of the Girsanov technique to the Lamperti factorization approach for explicitly computing the Laplace transforms $F_{G Y, a}$ with $a>0$.

21. Third proof of the theorem - a uniform argument. This section sketches a uniform way for computing the Laplace transforms $F_{G Y, a}$ with $a$ any positive real, and thus provides a third proof of $\S 8$ Theorem. Recall these transforms are defined by

$$
F_{G Y, a}(z)=\int_{0}^{\infty} e^{-z x} f_{G Y, a}(x) d x
$$

for any complex $z$ with $\operatorname{Re}(z)$ sufficiently big, and where $f_{G Y, a}(x)=$ $\mathbf{E}^{\mathbf{Q}}\left[f\left(A^{(\nu)}(x)\right)\right]$ setting $f(x)=(x-a)^{+}$. The key idea is to apply Girsanov's theorem such that $W_{x}=\nu x+B_{x}$ becomes a Brownian motion, and suppressing reference to this new measure, transcribe $f_{G Y, a}$ in terms of Yor's zero drift process $A^{(0)}$ as follows $f_{G Y, a}(x)=\mathbf{E}\left[f\left(A_{x}^{(0)}\right) e^{-\nu^{2} x / 2+\nu W_{x}}\right]$. With $\tau_{0, a}=\inf \left\{u \mid A^{(0)}(u)>0\right\}$ the inverse time change to $A^{(0)}$. at time zero, the computations of $\S 10$ thus prove $f_{G Y, a}(x)=0$ on the set of all events where $x \leqslant \tau_{0, a}$. And on the set of all events, where $x \geqslant \tau_{0, a}$ we now have the representation

$$
f\left(A^{(0)}\right)=\left(R_{a}^{(0)}\right)^{2} A_{x-\tau_{0, a}}^{(0)},
$$

which applies to express $f_{G Y, a}(x)$ as the following iterated expectation

$$
f_{G Y, a}(x)=\mathbf{E}\left[\left(R_{a}^{(0)}\right)^{2} \mathbf{E}\left[A_{x-\tau_{0, a}}^{(0)} e^{-\nu^{2} x / 2+\nu W_{x}} \mid \mathscr{F}_{\tau_{0, a}}\right]\right] .
$$

Applying Laplace transforms to both sides of this identity then gives

$$
\begin{aligned}
& F_{G Y, a}(z) \\
& \quad=\mathbf{E}\left[\left(R_{a}^{(0)}\right)^{2} e^{-\left(z+\nu^{2} / 2\right) \tau_{0, a}} \mathbf{E}\left[\int_{0}^{\infty} e^{-z x} A_{x}^{(0)} e^{-\nu^{2} x / 2+\nu W_{x+\tau_{0}, a}} d x \mid \mathscr{F}_{\tau_{0, a}}\right]\right] .
\end{aligned}
$$


In the conditional expectation restart the Brownian motion at time $\tau_{0, a}$. To the resulting additional $\nu$ th power of $\exp \left(W_{\tau_{0}, a}\right)$ apply the corresponding Lamperti factorization and interpret it as being equal to the $\nu$ th power of the zero index Bessel process at time $a$. Collecting powers of this last process,

$F_{G Y, a}(z)=\mathbf{E}\left[\left(R_{a}^{(0)}\right)^{\nu+2} e^{-\left(z+\nu^{2} / 2\right) \tau_{0, a}} \mathbf{E}\left[\int_{0}^{\infty} e^{-z x} A_{x}^{(0)} e^{-\nu^{2} x / 2+\nu W_{x}} d x \mid \mathscr{F}_{\tau_{0, a}}\right]\right]$.

The Strong Markov condition which we have used here also makes the whole integrand in the conditional expectation independent of time- $\tau_{0, a}$ information. Reversing the Girsanov transformation in this inner expectation,

$$
F_{G Y, a}(z)=\mathbf{E}\left[\left(R_{a}^{(0)}\right)^{\nu+2} e^{-\left(z+\nu^{2} / 2\right) \tau_{0, a}} \mathbf{E}\left[\int_{0}^{\infty} e^{-\left(z+\nu^{2} / 2\right) x} A_{x}^{(\nu)} d x\right]\right] .
$$

This puts us into the situation of $\S 10$. Partially reversing the Tonelli argument, the inner expectation is equal to the first moment of the drift- $\nu$ process $A^{(\nu)}$ at time $x$ recalled in $\S 4$ Lemma. Computing the Laplace transform, we thus arrive at

$$
F_{G Y, a}(z)=\frac{1}{2(\nu+1)}\left(\frac{1}{1-2(\nu+1)}-\frac{1}{z}\right) \mathbf{E}\left[\left(R_{a}^{(0)}\right)^{\nu+2} e^{-\left(z+\nu^{2} / 2\right) \tau_{0, a}}\right]
$$

if $\operatorname{Re}(z)$ is bigger than at least $\max \{0,2(\nu+1)\}$. In this way we are reduced to compute the expectation factor. This, however, proceeds as in $\S 10$ by conditioning on the corresponding Bessel process. The whole point is that this time conditioning is not on a Bessel process of an arbitrary index but on a Bessel processes of index zero. Observing how $\nu$ enters now via the exponent of the index zero Bessel process and via a shifting factor for the time change $\tau_{0, a}$, we so arrive at

$$
\mathbf{E}\left[\left(R_{a}^{(0)}\right)^{\nu+2} e^{-\left(z+\nu^{2} / 2\right) \tau_{0, a}}\right]=\frac{e^{-1 /(2 a)}}{a} \int_{0}^{\infty} e^{-x^{2} /(2 a)} x^{\nu+3} I_{\sqrt{2 z+\nu^{2}}}\left(\frac{x}{a}\right) d x,
$$

for any $z$ with sufficiently big positive real part. Using $\S 11$ Proposition, this integral is finite if $\operatorname{Re}(z)$ is positive and bigger than $2(\nu+1)$. So this third proof of $\S 8$ Theorem is complete.

\section{Part VII. Epilogue}

22. Consequences for Asian options - Hermite functions. Having persevered to this point, the reader may wonder about the nature and the quality of the implications of the mathematics developed up to now. Indeed, going back to the starting point of the journey, it seems that the Laplace transform approach makes possible significant improvements in understanding $\S 3$ 's normalized prices $C^{(\nu)}(h, q)$ of Asian options themselves. These improvements are on a structural level and as such make possible advances on computing $C^{(\nu)}$ as a consequence. This is essentially by being able 
to establish new links of Asian option valuation with a well-studied class of special functions: the Hermite functions to be reviewed in this section.

Following [15, $\S 10.2 \mathrm{ff}]$, to which we refer for details, Hermite functions $H_{\mu}$ are analytic on the complex plane as functions of both their variable $z$ and their degree $\mu$. If the real part $\operatorname{Re}(\mu)$ of $\mu$ is bigger than -1 , they have the integral representation

$$
H_{\mu}(z)=\frac{2^{\mu+1}}{\sqrt{\pi}} e^{z^{2}} \int_{0}^{\infty} e^{-x^{2}} x^{\mu} \cos \left(2 z x-\frac{1}{2} \mu \pi\right) d x
$$

So they specialize to the $\mu$ th Hermite polynomials if $\mu$ is any nonnegative integer, whence $H_{0}=1, H_{1}(z)=2 z, H_{2}(z)=4 z^{2}-2, H_{3}(z)=8 z^{3}-12 z$, for example. If the real part of $\mu$ is negative, however, Hermite functions change their character. Then they have the integral representation:

$$
H_{\mu}(z)=\frac{1}{\Gamma(-\mu)} \int_{0}^{\infty} e^{-u^{2}-2 z u} u^{-(\mu+1)} d u
$$

and so specialize via $(2 / \sqrt{\pi}) H_{-1}(z)=\exp \left(z^{2}\right) \operatorname{Erfc}(z)$ to the complementary error function Erfc recalled to be given by

$$
\operatorname{Erfc}(z)=\frac{2}{\sqrt{\pi}} \int_{z}^{\infty} e^{-\xi^{2}} d \xi
$$

For any complex $\mu$, Hermite functions can be expressed in terms of the Kummer confluent hypergeometric function $\Phi$ by

$$
H_{\mu}(z)=\frac{2^{\mu} \Gamma(1 / 2)}{\Gamma((1-\mu) / 2)} \Phi\left(-\frac{\mu}{2}, \frac{1}{2} ; z^{2}\right)+z \frac{2^{\mu} \Gamma(-1 / 2)}{\Gamma(-\mu / 2)} \Phi\left(\frac{1-\mu}{2}, \frac{3}{2} ; z^{2}\right)
$$

for any complex $z$. From this representation one can moreover see how Hermite functions are connected with the parabolic cylinder functions $D_{\mu}$ and with the Kummer confluent hypergeometric function of the second kind $\Psi$.

23. Consequences for Asian options - new integral representations. Hermite functions as recalled in the previous section appear naturally in the closed form solution for $\S 3$ 's normalized price $C^{(\nu)}(h, q)$ of the Asian option we have developed in [21]. If the normalized strike price $q$ is positive, it expresses this value as the sum of integral representations which have a product structure. They are obtained by integrating products of Hermite functions $H_{\mu}$ with weighted error functions as follows.

Theorem. If $q$ is positive, the normalized price $C^{(\nu)}(h, q)$ of the Asian option is given by the following difference

$$
C^{(\nu)}(h, q)=c e^{2 h(\nu+1)} S_{\nu+2}-c S_{\nu},
$$

where the $S_{\xi}$ are three-term sums $S_{\xi}=C_{\text {trig, },}\left(\rho_{\xi}\right)+C_{\text {hyp }, \xi}\left(\rho_{\xi}\right)+C_{\text {hyp },-\xi}\left(\rho_{\xi}\right)$ whose single summands are integrals that depend on parameters $\rho_{\xi} \geqslant 0$, but which as a whole are independent of these. 
In terms of $\S 3$ 's concepts, $c$ is given by

$$
c=c(\nu, q)=\frac{\Gamma(\nu+4)(2 q)^{(\nu+2) / 2}}{2 \pi(\nu+1) e^{1 /(2 q)}}
$$

recalling $\nu=2 \varpi / \sigma^{2}-1$. With $\rho$ any nonnegative real, the trigonometric terms $C_{\text {trig, } \xi}(\rho)$ with $\xi$ equal to $\nu$ or $\nu+2$ are the integrals

$$
C_{\mathrm{trig}, \xi}(\rho)=\int_{0}^{\pi / 2} \operatorname{Re}\left(H_{-(\nu+4)}\left(-\frac{\operatorname{ch}(\rho+i \phi)}{\sqrt{2 q}}\right) E_{\xi}(h)(\rho+i \phi)\right) d \phi
$$

over the real parts of products of Hermite functions times certain functions $E_{b}(h)$, and the hyperbolic terms $C_{\mathrm{hyp}, \xi}(\rho)$ with $\xi$ equal to $\pm \nu$ or $\pm(\nu+2)$ are the integrals

$$
C_{\mathrm{hyp}, \xi}(\rho)=\int_{\rho}^{\infty} \operatorname{Im}\left(H_{-(\nu+4)}\left(-\frac{\operatorname{sh}(y)}{\sqrt{2 q}} i\right) E_{\xi}(h)\left(y+i \frac{\pi}{2}\right)\right) d y
$$

over the imaginary parts of such products. Herein $E_{\xi}(h)$ are the weighted complementary error functions for any complex $w$ given by

$$
E_{\xi}(h)(w)=e^{w \xi} \operatorname{Erfc}\left(\frac{w}{\sqrt{2 h}}+\frac{\xi}{2} \sqrt{2 h}\right) .
$$

$\mathrm{R} \mathrm{e} \mathrm{m}$ a $\mathrm{rk}$. If $\rho$ equals zero, the trigonometric terms specialize to

$$
C_{\text {trig }, \xi}(0)=2 \int_{0}^{\pi / 2} H_{-(\nu+4)}\left(-\frac{\cos (\phi)}{\sqrt{2 q}}\right) \cos (\xi \phi) d \phi .
$$

Comparing with the formula of $\S 5$, the above formula, is given as a sum of single integrals whose integrands have a structural interpretation as products of two functions. It identifies the higher transcendental functions occurring as factors in these products, and shows how they are given by or built up from Hermite functions.

24. Epilogue. On a technical level, the differences just noted between $\S 5$ 's and $\S 23$ 's formula can be regarded as consequences of the different mathematical approaches for proving the valuation formula. In fact, $\S 5$ 's originates from Yor's direct attack on the law of the integral of geometric Brownian motion. In contrast, $\S 23$ 's is eventually based on the indirect enveloping construction of $\S 7$. In fact, to obtain Asian option prices, first analytically invert $\S 8$ Theorem's Laplace transforms $F_{G Y, a}$, then proceed using the key reduction of $\S 7$ Lemma; formally speaking: $C^{(\nu)}(h, q)=\mathscr{L}^{-1}\left(F_{G Y, q}\right)(h)$.

However, there are not only structural differences between $\S 5$ 's and $\S 23$ 's formula. Finally deriving benchmarks for the normalized prices $C^{(\nu)}(h, q)$ of Asian options seems to be one of the main practical application of such formulas. As we have already mentioned, Yor's formula seems 
impracticable for this purpose in particular because of the gigantic size of the numbers it involves. For instance, consider valuing Asian options with annual interest rates $r$ equal to nine percent, with maturities of one year, with $K=S_{0}$, with $t_{0}=0$, and with a volatility of $\sigma=30 \%$. These values require coping with numbers of order $10^{100}$. Our formula improves on this aspect as well. In the above situation, for instance, the four hyperbolic terms have orders $10^{7}$ and the two trigonometric terms have orders $10^{-2}$. Sharpening results of [20], it thus became possible to derive in [23, Chapter 5] the following first time benchmark values for normalized Asian option prices:

\begin{tabular}{|c|c|c|}
\hline$\sigma$ & maximalerror & $C^{(\nu)}(h, q)$ \\
\hline $20 \%$ & $4.9727 \times 10^{-16}$ & 0.00074155998788343 \\
\hline $30 \%$ & $4.9687 \times 10^{-16}$ & 0.00217354504625037 \\
\hline $40 \%$ & $4.9157 \times 10^{-16}$ & 0.00478100328341654 \\
\hline $50 \%$ & $4.9461 \times 10^{-16}$ & 0.00890942045213227 \\
\hline
\end{tabular}

Table 3. Normalized prices $C^{(\nu)}(h, q)$ of the Asian option for $T=1$.

All of this may be seen as a consequence of the Laplace transform approach to valuing non-Asian options initiated in [12]. With hindsight, this paper appears to be a rich source for new results in both mathematics and finance.

Acknowledgments. The second author is grateful to the support by the Deutsche Forschungsgemeinschaft and the hospitality of the Mathematisches Institut of the Universität Mannheim. While the usual disclaimer applies, we would also like to thank the editor Professor A.N. Shiryaev for his interest in the paper and the efforts he has spent on it.

\section{REFERENCES}

1. Beals R. Advanced Mathematical Analysis. Berlin-Heidelberg: Springer-Verlag, 1973, $230 \mathrm{p}$.

2. Carr $P$., Schröder $M$. On the valuation of arithmetic-average Asian options: the Geman-Yor Laplace transform revisited. - Mannheim and New York, December 2000; http://arXiv.org/abs/math.CA/0102080.

3. Conway J.B. Functions of One Complex Variable. Berlin-Heidelberg: SpringerVerlag, 1984.

4. Doetsch G. Handbuch der Laplace Transformation. V. I. Basel: Birkhäuser, 1971, $581 \mathrm{p}$.

5. Donati-Martin C., Ghomrasni R., Yor M. On certain Markov processes attached to exponential functionals of Brownian motion: application to Asian options. - Rev. Mat. Iberoamericana, 2001, v. 17, № 1, p. 179-193.

6. Duffie $D$. Security Markets. Boston: Academic Press, 1988,358 p.

7. Duffie D. Dynamic Asset Pricing Theory. Princeton: Princeton Univ. Press, 1996.

8. Dufresne $D$. The distribution of a perpetuity, with applications to risk theory and pension funding. - Scand. Actuar. J., 1990, № 1-2, p. 39-79.

9. Dufresne D. Laguerre series for Asian and other options. - Math. Finance, 2000, v. 10 , № 4 , p. 407-428. 
10. Freitag E., Busam R. Funktionentheorie. Berlin: Springer-Verlag, 1993, 473 p.

11. Fu M. C., Madan D. B., Wang T. Pricing continuous Asian options: a comparison of Monte Carlo and Laplace inversion methods. - J. Comput. Fin., 1998, v. 2, p. 49-74.

12. Geman H., Yor M. Bessel processes, Asian options, and perpetuities. - Math. Finance, 1993, v. 3, № 4, p. 349-375.

13. Karatzas I., Shreve S.E. Methods of Mathematical Finance. New York: SpringerVerlag, 1998, $407 \mathrm{p}$.

14. Knight F. B. Essentials of Brownian Motion and Diffusion. Providence, RI: Amer. Math. Soc., 1991.

15. Лебедев Н.Н. Специальные функции и их применение. М.: Физматгиз, 1968, $324 \mathrm{c}$.

16. Musiela M., Rutkowski M. Martingale Methods in Financial Modelling. Berlin: Springer-Verlag, 1997, $512 \mathrm{p}$.

17. Øksendal B. Stochastic Differential Equations. Berlin: Springer-Verlag, 1998, 324 p.

18. Rudin W. Real and Complex Analysis. New York: McGraw Hill, 1987, 416 p.

19. Revuz D., Yor M. Continuous Martingales and Brownian Motion. Berlin: SpringerVerlag, 1994, $560 \mathrm{p}$.

20. Rogers L. C. G., Shi Z. The value of an Asian option. - J. Appl. Probab., 1995, v. 32, p. $1077-1088$.

21. Schröder $M$. On the valuation of arithmetic-average Asian options: integral representations. Preprint. Mannheim: Universität Mannheim, Oktober 1997, revised November 1999; http://arXiv.org/abs/math.CV/0003055.

22. Schröder $M$. On the valuation of arithmetic-average Asian options: explicit formulas. Preprint. Mannheim: Universität Mannheim, März 1999.

23. Schröder M. Mathematical ramifications of option valuation: the case of the Asian option. Habilitationsschrift. Universität Mannheim, April 2002.

24. Schröder $M$. On the valuation of arithmetic-average Asian options: Laguerre series and Theta integrals. Preprint. Mannheim: Universität Mannheim, Dezember 2000; http://arXiv.org/abs/math.CA/0012072.

25. Watson G.N. A treatise on the theory of Bessel functions. Cambridge: Cambridge Univ. Press, 1944, 804 p.

26. Weil A. Euvres scientifiques. V. 3 (1964-1974). Berlin-Heidelberg: Springer-Verlag, $1980,465 \mathrm{p}$.

27. Yor $M$. Loi d'indice du lacet Brownien, et distribution de Hartman-Watson. Z. Wahrscheinlichkeitstheor. verw. Geb. 1980 , v. 53, p. 71-95.

28. Yor $M$. Sur certaines fonctionnelles exponentielles du mouvement Brownien réel. J. Appl. Probab., 1992, v. 29, p. 202-208.

29. Yor $M$. On some exponential functionals of Brownian motion. - Adv. Appl. Probab., 1992 , v. 24, p. 509-531.

30. Yor M., Göing-Jaeschke A. A survey and some generalizations of Bessel processes. Zürich: ETH, 1999.

31. Yor M., Donati-Martin C., Matsumoto H. Exponential functionals of Brownian motion and related processes III. Preprint. Paris VI, May 2000.

32. Yor $M$. Exponential Functionals of Brownian Motion and Related Processes. Berlin: Springer-Verlag, 2001, $205 \mathrm{p}$. 